\title{
Where in the tropics are all of the specialist armored scale insects?
}

\author{
Daniel Peterson ${ }^{1}$, Nate Hardy ${ }^{2}$, Geoffrey Morse ${ }^{3}$, Takao Itioka ${ }^{4}$, Jiufeng Wei $^{5}$, and \\ Benjamin Normark 6 \\ ${ }^{1}$ University of Massachusetts System \\ ${ }^{2}$ Auburn University \\ ${ }^{3}$ University of San Diego \\ ${ }^{4}$ Kyoto University \\ ${ }^{5}$ Shanxi Agricultural University \\ ${ }^{6}$ University of Massachusetts Amherst
}

June 2, 2020

\begin{abstract}
Most herbivorous insects are diet specialists in spite of the apparent advantages of being a generalist. This conundrum might be explained by fitness trade-offs on alternative host plants, yet evidence of such trade-offs has been elusive. Another hypothesis is that specialization is non-adaptive, evolving through neutral population genetic processes and within the bounds of historical constraints. Here we report on a striking lack of evidence for the adaptiveness of specificity in tropical canopy communities of armored scale insects. We show that specialists abound and that host-use is phylogenetically conservative, but in comparison to generalists, specialists occur on fewer of their potential hosts, and are no more abundant where they do occur. Of course local communities might not reflect regional diversity patterns. But based on our samples, comprising hundreds of species of hosts and armored scale insects at two widely separated sites, host-use specialists do not appear to outperform generalists.
\end{abstract}

\section{Introduction}

High species diversity has been attributed to the partitioning of available resources into narrow ecological niches (Hutchinson 1959). Yet niche width varies greatly between species. Herbivorous insects are classic subjects for the study of this variation. Although diet specialists prevail, diet breadths vary continuously, and some generalists are extremely so (Normark and Johnson 2011; Forister et al. 2015). How did this come to be?

For the most part, theorists have worked from the premise that diet specialization comes from genetic tradeoffs between adaptations to alternative resources, specifically antagonistic pleiotropy between alleles at a few diet-determining loci (Futuyma and Moreno 1988; Ravigné et al. 2009). Although empirical evidence for such genetic trade-offs is scarce (Futuyma 2008; Forister et al. 2012), they might be difficult to detect, as they can be hidden by inter-individual fitness variation at linked loci (Joshi and Thompson 1995), or arise from epistatic interactions between alleles (Remold 2012; Rodriguez-Verdugo et al. 2014; Celorio-Mancera et al. 2016). In sum, the evidence is scarce for the adaptive trade-off hypothesis, but it is difficult to falsify outright.

Alternatively, niche specialization could be driven by non-adaptive processes (Futuyma et al. 1995; Gompert et al. 2015). In fact, theoretical spatial models have shown that adaptive trade-offs are not necessary to produce niche-breadth distributions resembling those observed in natural communities (Forister and Jenkins 2017). Evolving the ability to use a novel host almost certainly entails directional selection. But alleles promoting fitness on other potential hosts can be lost through genetic draft during strong directional selection 
on a novel host (Neher 2013), or genetic drift when insect and host distributions cease to overlap (Gompert et al. 2015). One way or another, if host-use traits are easy to lose but difficult to get back, neutral genetic processes could pull populations towards niche specialization (Hardy et al. 2016).

Host-use trade-offs in herbivorous insects have traditionally been investigated by comparing performance across multiple host plants of different insect genotypes within a population. But a phylogenetically-informed comparison of host-use across multiple herbivore species offers a complementary perspective that may be less obscured by short-term genetic contingencies (Funk et al. 1995; Futuyma 2010; Hardy and Otto 2014; Peterson et al. 2015, 2016). To wit, it could illuminate the overall relationship between diet-breadth and ecological performance. If host-use specificity is adaptive, we would expect that on any shared host specialists would tend to perform better than generalists. Likewise, at the meta-population level, if host-use specificity is adaptive, we might expect specialists to do a better job of colonizing specific host resources (Gryllenberg and Metz 2001). Conversely, if specificity is non-adaptive, we would expect generalists to colonize more of their potential hosts, and to perform just as well as specialists on shared hosts, or even perform better if there is a population-genetic cost for specificity, for example reduced population size and more erosive genetic drift.

We sought evidence of such performance differences in the relative abundances and patch occupancies of 171 putative armored scale insect species (Hemiptera: Diaspididae) across 138 tree species in tropical rainforest communities on two continents. Diaspidids are sessile and have a simple, pathogen-like life history in which new host trees are colonized by wind-dispersed first-instar nymphs that cannot survive for long away from a host (Hardy 2018). Potential for host-choice is therefore limited and occurrence of non-dispersive life stages on a plant is a relatively clear indication that it is a suitable host for development and reproduction (Hill and Holmes 2009). With random, time-limited dispersal, one might expect the greatest fitness for genotypes that perform best across most of the commonly encountered host-plants. In fact, for diaspidids, we have previously shown that when host associations are treated as a binary use-or-nonuse traits, the phylogenetic patterns of host use are incompatible with strong adaptive trade-offs (Peterson et al. 2015). Nevertheless, we have not previously been able to account for potentially important quantitative differences in performance across host plant groups.

Our approach was to (1) estimate allele genealogies among the sampled diaspidids for 3 loci, using DNA sequence data; (2) estimate species boundaries using these genealogies and also using morphology; (3) estimate the degree to which host use is phylogenetically conservative; (4) explicitly test for diet specialization in each species; and (5) use abundance-based and patch-occupancy-based indices of performance to test if specialists tend to do better than generalists on shared hosts.

\section{Methods}

What to measure? Diet-breadth dependent performance trade-offs could result from any number of mechanistic interactions between a herbivorous insect and a host plant. On a particular host plant, in comparison to a specialist, a generalist might have (1) a reduced ability to initiate feeding, (2) a lower feeding rate, (3) less efficient utilization of host nutrients, (4) greater susceptibility to host defenses, or (5) more exposure to natural enemies. No matter the mechanism, any trade-offs that drive the evolution of specialization would need to ultimately limit survival or fecundity. If specialization is an adaptive response to trade-offs between performance on alternative hosts, specialists should have higher survival or fecundity than generalists on shared resources. In the studied tropical forest plots, we were not able to measure survival or fecundity directly, but we were able to measure the abundance and patch occupancy of each diaspidid species on each host-plant species. As mentioned earlier, because after the crawler stage each diaspidid is stuck for life on one host, an observation of a second-instar or adult individual on a host is evidence of successful development on the host (Hill and Holmes 2009). Moreover, the relative abundance of diaspidid species on each host-plant species is an especially synthetic proxy for fitness - integrating across host-dependent differences in diaspidid fecundity and survival.

Sampling. We surveyed diaspidids at two tropical rainforest sites: (1) San Lorenzo National Park, Panama 
and (2) Lambir Hills National Park, Malaysia (on the island of Borneo). At each site, we used a crane to reach the forest canopy. We were not able to search each tree in each plot, so we used preexisting databases of the trees at each site to divide identified individual trees into sampling groups of one randomly-selected individual per tree species. Only trees over $10 \mathrm{~cm}$ diameter at breast height were considered. We did not sample any tree individual more than once, so tree species with only one individual were present only in the first round of sampling, those with two individuals were present in the first two rounds, and so on. This protocol allowed us to sample across the full diversity of host taxa while also getting multiple samples from common host species.

At each focal tree, 20 person-minutes were allocated to visual searching of accessible foliage. Any leaves and twigs that we saw were infested by diaspidids, we cut from the tree and collected. From each tree we also haphazardly took one $20 \mathrm{~cm}$ twig sample and one $20 \mathrm{~cm}^{2}$ bark sample. Removed plant material was stored in plastic bags and transferred to the lab for processing under magnification; live diaspidids were cut from the surrounding plant material and preserved in $95 \%$ ethanol. Specimens were subsequently sorted to life stage and second-instars and adult females were regarded as evidence of successful establishment.

Phylogenetics. DNA was extracted from all second-instar and adult females and using Qiagen DNeasy Blood \& Tissue kits (Qiagen, Valencia, CA) following the procedure outlined in Normark et al. (2014). We amplified three loci that have previously been used for diaspidid phylogenetics: elongation factor 1- $\alpha$ (EF1 $1 \alpha)$, part of the large ribosomal subunit rDNA gene (28S), and a part of the mitochondrial genome spanning cytochrome c oxidase I and II (COI-II). PCR primers and protocols followed Andersen et al. (2010) and Gwiazdowski et al. (2011). PCR products were visualized using 1.5\% agarose gels with SYBRsafe (Invitrogen, Carlsbad, CA, USA) and successful reactions were purified with Exo SAP-IT enzymatic digestion (Affymetrix, Cleveland, OH, USA). Sanger sequencing of the PCR products was completed by Macrogen (Cambridge, MA, USA) or Eton Biosciences (San Diego, CA, USA). Genbank Accessions are provided in S1.

Phylogenetic relationships among all sampled individuals were estimated from the DNA sequence data. Sequences from each genetic locus were aligned using PASTA (Mirarab et al. 2014), and alignments were trimmed to include only sites with non-gap sequence for at least $80 \%$ of specimens (Capella-Gutiérrez et al. 2009). Genealogies were inferred using the GTR+CAT model in RAxML (Stamatakis 2014). The three single-locus alignments were then combined as one supermatrix, from which we also inferred a phylogeny with RAxML. For use in comparative analyses, we made a version of the phylogeny with just one tip per species, and scaled branch lengths to time using an auto-correlated model of among-lineage rate variation, fit with penalized likelihood as implemented in treePL (Smith and O'Meara 2012), and constraining the armored scale root to be 50-75 million years old (Vea and Grimaldi 2016).

Species Delimitation and Identification. We delimited putative species with a version of the genealogical concordance method (as in Gwiazdowski et al. 2011). All clades shared by at least two gene trees, and not contradicted by the third gene tree, were considered evolutionarily independent lineages. Species were defined provisionally as the most inclusive independent lineages containing at least three terminal branches and no more exclusive independent lineages. This method precludes delimitation of species represented by fewer than three specimens. To work around this problem, we calculated the minimum divergence between provisional species clades, and used that value as a maximum threshold for within-species divergence. Any specimens separated by more than this distance from all other specimens were also considered distinct species. We also examined specimens and identified them according to standard morphological criteria to the extent that this was possible. Because second instars and adults were both included in this study, whereas standard keys and descriptions are based on adults only, direct morphological comparisons and identifications were not always possible. The analyses below were repeated for DNA-based and morphology-based species delimitations. We retained all specimens in both analyses, whether or not they were morphologically identifiable; for a few specimens that were not morphologically identifiable, in the morphology-based analysis we defaulted to the DNA-based species.

Statistical Analysis. We characterized host-use specialization by diaspidid species in two ways, each applied at three levels of host plant taxonomy (species, genus, and family). First, we quantified diet specificity; we asked 
whether diaspidids used less diverse hosts than expected by chance. Concretely, for each diaspidid species, we quantified host-taxon diversity using Simpson's Reciprocal Diversity Index (RDI), which is essentially evenness-corrected host-taxon richness. We compared empirical RDIs to those expected under a null model of random host use. We simulated 1000 null data sets by randomly permuting the associations between diaspidid species and individual host trees; then for each permutation, we again calculated the mean RDI for the hosts of each diaspidid. With this approach, a diaspdid species is a specialist if its host RDI is lower than expected under the null model.

In a second view of host-use specialization, we calculated the phylogenetic conservatism of host use across diaspidid species. In other words, we asked if evolutionary history constrains host use. We used the R package (R Core Team 2017) MCMCglmm (Hadfield and Nakagawa 2010) to measure the phylogenetic signal of host use by estimating the proportion of variance in the binary use-or-non-use of each host taxon that could be explained by the diaspidid phylogeny. Empirical values for phylogenetic signal were then compared to those calculated under a null model. Null data sets were produced by randomly swapping associations between diaspidid species and host taxa until the associations were thoroughly shuffled (the number of random swaps was 10 times the overall number of associations). This preserved the empirical distribution of diet breadths while randomizing specific associations. $P$ - values for the empirical phylogenetic signal values were calculated using a $Z$-test against each parameter's null data set values (which were approximately normally distributed). We corrected for multiple comparisons by assigning statistical significance according to a false discovery rate (FDR; Benjamini \& Hochberg 1995) of 0.05. The FDR procedure was conducted separately for each host-taxon level because these analyses were not independent, and must be interpreted as alternative configurations of the same data.

We investigated the strength of performance trade-offs by calculating for each host tree taxon the correlation between diaspidid diet breadth (count of host taxa) and mean abundance. If performance trade-offs are strong, on any given host taxon, we expect generalist diaspidids to be less abundant than specialists. We also investigated the relationship between diet breadth and the proportion of host trees of a taxon colonized at each site, as patch occupancy may be a better indicator of fitness than local abundance in a metapopulation of discrete colonies (Gyllenberg and Metz 2001). Using R, we fit generalized linear models. For local abundance, the response variable was the number of diaspidid individuals identified per host tree, assuming a Poisson distribution. For metapopulation colonization-rate, the response variable was the probability that an individual tree within each host taxon would be colonized by a diaspidid species, assuming a binomial distribution and excluding host taxa with fewer than 3 trees surveyed. Both models only incorporated data for host-taxon-by-diaspidid associations with at least one record. To assess statistical significance, we compared empirical coefficients to those estimated from 1000 null data sets, produced by randomly permuting the empirical data.

The scripts used for the analysis will be made available at Dryad.

\section{Results}

DNA-based species delimitations. In Panama we surveyed 90 trees over three rounds of sampling, representing 53 species, 48 genera, and 29 families. We found live diaspidids on 75 trees, yielding 380 female specimens (adults and second instars). At least two loci were successfully amplified for 184 specimens, belonging to 53 DNA-delimited species (Fig. A1.6; Table A1.1). Assignment to a morphologically defined species was possible for 180 specimens, representing 32 described and 12 undescribed species. Species assignments and trophic links are in Table S2. In Malaysia, we surveyed 211 trees over 20 rounds of sampling, including 85 species, 48 genera, and 27 families. We found live diaspidids on 102 trees, yielding 480 female specimens. At least two loci were successfully amplified for 266 specimens, belonging to 123 DNA-delimited species (Fig. A1.7 Table A1.1). Assignment to a morphologically defined species was possible for 259 specimens, representing 20 described and 58 undescribed species. Species assignments and trophic links are in Table S3.

We found strong evidence for host-use specialization, both in terms of less-than-expected host plant diversity, and more-than-expected phylogenetic conservatism of host use. The Simpson's RDI of each diaspidid species' 
diet was significantly lower than expected at all host taxonomic levels and in both locations, except at the host-species level in Panama (Table A1.2). Phylogenetic signal was significantly stronger than its null expectation for 19 host taxa (Figure A1.8), although it was higher at the Malaysia site (mean 0.61) than the Panama site (mean 0.45), with 18 Malaysian host taxa with significant phylogenetic conservatism, compared to just one Panamanian host taxon with significant conservatism.

Despite the prevalence of diaspidid host-use specialization at our two sites, and of extensive phylogenetic conservatism of host use in Malaysia, we found no evidence for performance trade-offs on alternative hosts that would select against broad diets. Specialists were no more abundant than generalists on specific host trees; the number of live adult or second instar female diaspidids found on each tree was not correlated with diet breadth (Fig. 1; Table A1.4). Moreover, contra the metapopulation trade-off hypothesis, diaspidids with broader diets were observed on a higher proportion of the trees in their host taxa, although this effect was not significant for Malaysian diaspidids and their host plant species (Figure 2; Table A1.4).

Morphology-based delimitations. The results of the analyses using morphologically delimited species were broadly consistent with those using DNA-delimited species. As for analyses using DNA-delimited species, with morphology-delimited species we found that diaspidid species were more specialized than expected by chance (Table A1.3), that more specialized species were no more abundant on their hosts (Table A1.5), and tended to occupy a smaller proportion of their potential host plants (Table A1.5). Actually, when modelling the links between Malaysian diaspidids and their hosts at the species level, we found positive effect of diet breadth on abundance that fell just short of significance $(\mathrm{p}$-value $=0.08)$.

\section{Discussion}

Diaspidid species in tropical rainforest canopy habitats appear to use as hosts only a small proportion of the plant taxa in their local environment; simply put, diaspidids tend to be diet specialists. But across the hundreds of trees that we surveyed, more specialized species were no more abundant on their hosts than generalists, and occurred on a smaller proportion of their potential host plants. Is specialization for these diaspidids non-adaptive?

Such a conclusion would hinge on the assumption that what we saw within the reach of canopy cranes is what we would have seen elsewhere. But if abundance varies much over space, local differences in abundance could be misleading. Some such heterogeneity is expected. The quality of specific host-plant resources can vary due to spatial mosaics of natural enemy pressure (Heard et al. 2006), as well as host-plant features such as genotype, induced defensive state, and physical structure (Dixon 2005). Although we saw no abundant specialist species, each could have been abundant somewhere else in the forest, where more suitable resources occur. Nevertheless, extreme patchiness in the abundance of specialists would entail a meta-population fitness cost, as local catastrophes would be more likely to cause extinction (Nurmi \& Parvinen 2008). In sum, potential spatial variation in abundance keeps us from making definite conclusions about the adaptiveness of specificity in diaspidids. But this potential is diminished by the cost of meta-population patchiness, and the consistency of our observations across species and communities.

We found that the use of many host taxa by diaspidids was phylogenetically conservative. Although such conservatism of host use has been found for several other groups of herbivorous insects, such as butterflies (Janz et al. 2001) and beetles (Kelley and Farrell 1998), it has a special significance for diaspidids, as they colonize new hosts haphazardly via wind (Magsig-Castillo et al. 2010). Consequently, it may more likely denote historical constraints on contemporary niches than long-term persistence of niche optimization. In our previous work, we found no evidence for performance trade-offs for diaspidids between alternative hosts (Peterson et al. 2015); thus host-use constraints would seem to persist in the face of what may be strong selection for broad diets.

Our results also shed light on the complexity of host-use traits in plant-feeding insects (Barrett and Heil 2012; Forister et al. 2012). We found that specialization in armored scale insects occurs at all three of the hosttaxonomic levels that we considered (species, genus, and family), suggesting that the genomic architecture of host-use traits is both complex and hierarchical. Use of multiple hosts is often associated with close 
phylogenetic relationships among those hosts (Gilbert and Webb 2007; Krasnov et al. 2012), yet such results in flying insects may reflect host-preference or ease of host recognition more than host performance (Bernays 2001). Because diaspidids have little opportunity to choose a host, phylogenetic conservatism at multiple taxonomic levels implies that performance on a host likely depends on many traits of various effect sizes. Although actual mechanisms are as yet unclear (but see Hogenhout and Bos 2011; Ali and Agrawal 2012), the involvement of many genetic loci in plant-insect interactions is consistent with both ecological (Singer and Stireman 2005) and genetic (Remold 2012) theory as well as recent genome-wide association studies (e.g., Egan et al. 2015; Gompter et al. 2015).

Our DNA-based species delimitation allow us some insight into whether any species that have been characterized as extremely polyphagous (Normark \& Johnson 2011; Normark et al. 2014) are in fact clusters of cryptic specialists. The answer is mixed. On the one hand, in Panama, the single most polyphagous species in the sample, Selenaspidus articulatus (Morgan) shows no hint of cryptic species diversity - not surprisingly, as it is native to Africa and invasive in Panama (Normark et al. 2019). On the other hand, several other reportedly highly polyphagous species do appear to represent cryptic species clusters. In Panama, only a single morphologically-delimited species shows evidence of cryptic diversity: samples of Diaspis boisduvalii (Signoret) were apportioned across five DNA-delimited species. In contrast, at the Malaysian site, cryptic diversity appears rampant, especially among the most polyphagous species:Chrysomphalus dictyospermi (Morgan), purported to use 80 host families worldwide, was recovered as two cryptic species; Chrysomphalus pinnulifer (Maskell), with 40 host families worldwide, was also recovered as two cryptic species; Morganella longispina (Morgan), 22 host families worldwide, three cryptic species;Aonidiella inornata McKenzie, 24 host families worldwide, three cryptic species. We also found cryptic diversity in less polyphagous Southeast Asian species: Silvestraspis uberifera (Lindinger), three cryptic species, and Aulacaspis calcarata (Takagi), eight cryptic species, as well as in several undescribed species. Most strikingly, one undescribed species provisionally designatedSishanaspis ud4977 appears to comprise a complex of 10 cryptic species. The upshot is that in Malaysia traditional morphology-based species delimitation seems to miss much of the true diversity. But our inferences about the extent and consequence of diet specificity in diaspidids appear robust to how species are delimitated.

One other insight afforded by the morphological species identifications, which may help explain difference in diet breadth and host occupancy observed between the two sites, is the incidence of invasive species. At the Malaysian site we found no genera native to regions other than Southeast Asia, whereas in Panama nearly half of morphologically identifiable individuals $(77 / 180=43 \%)$ belong to invasive species (Normark et al. 2019). In addition to Selenaspidus articulatus(sampled on 18 host species), these include several genetically uniform populations that we sampled on multiple host species, includingLepidosaphes rubrovittata (Cockerell) (6 hosts), Chrysomphalus dictyospermi (4 hosts), Aspidiotus excisusGreen (3 hosts), and Lepidosaphes punicae Laing (3 hosts). Thus the narrower diets and higher host occupancy in Panama could have something to do with the relatively recent arrival of much of the diaspidid fauna.

In conclusion, evolutionary fitness is notoriously difficult to measure. We can not draw straight lines connecting it to differences in local abundance and patch occupancy. It could be that for diaspidids the quality of host resources is extremely uneven across tropical canopies, and that for each of the specialists we sampled there was an unsampled population booming somewhere else in the forest. Or it could simply be that host specialization is not adaptive for wind-dispersed plant pathogens in diverse host-plant communities. If hostuse specialization is adaptive and high quality hosts are patchy and rare, then the question becomes this: where in the forest are all the specialists? What are the conditions that must be met for a specialist to make good on their specialty?

\section{Acknowledgements}

We thank the Smithsonian Tropical Research Institute for facilitating our fieldwork in Panama. Our field survey at Lambir Hills National Park was conducted in accordance with the Memorandums of Understanding signed between the Sarawak Forest Department (SFD, Kuching, Malaysia) and the Japan Research Consortium for Tropical Forests in Sarawak (JRCTS, Sendai, Japan) in December 2012. Thanks to Mohd. Shahbudin 
Sabki, Engkamat Lading, and Mohamad bin Kohdi, Paulus Meleng of SFD for help in obtaining research permission at the Lambir Hills National Park. We thank Tohru Nakashizuka (Tohoku University, Sendai, Japan) and Tomoaki Ichie (Kochi University, Kochi, Japan) for their support. Diaspidid sample processing was by Katelyn Mullen, Ryan McCarthy, Mitchel Logan, Michael Fizdale, Kyara Romeu, Shannon Trujillo, Hannah Shapiro, and Anna Whitfield. DAP's dissertation committee (Michael Hood, Adam Porter and Laura A. Katz) and anonymous reviewers provided helpful feedback. JW's participation was supported by the China Scholarship Council. This work was supported by the National Science Foundation (DEB-1258001; DEB-1744552) and by Grants-in-Aid (no. 21255004 to TI) from the Japanese Ministry of Education, Science and Culture.

Figures
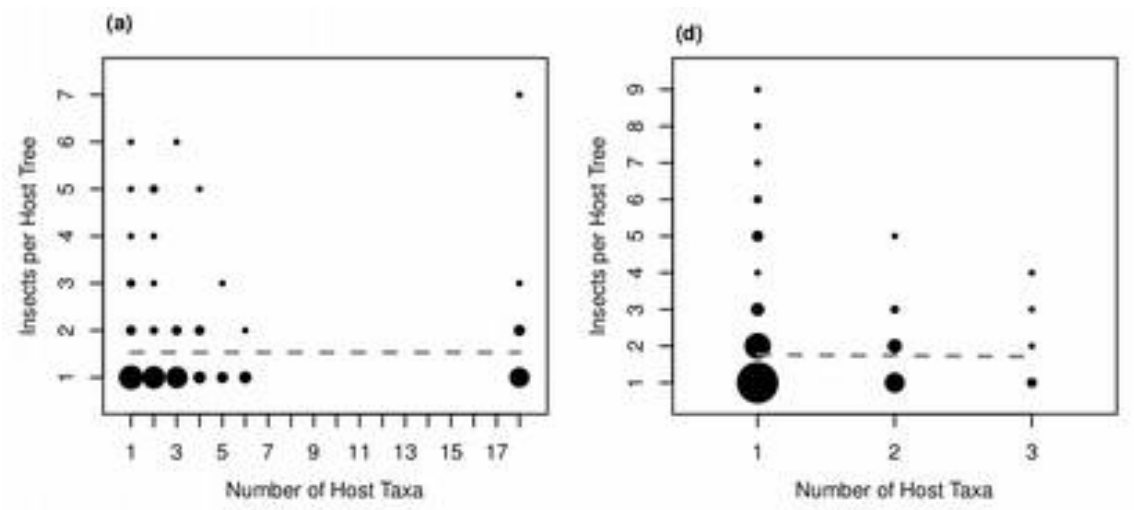

(b)
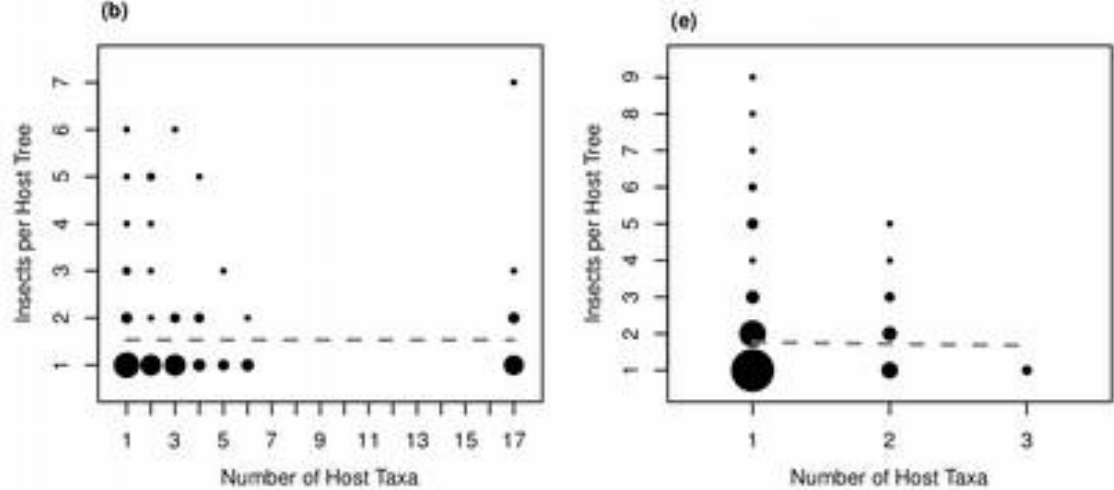

(c)
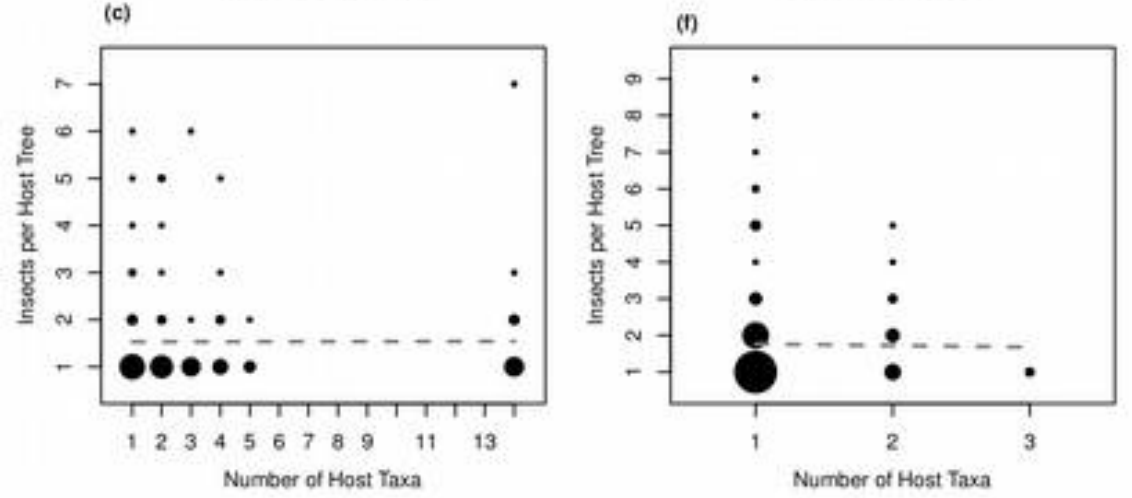

Figure 1. We found no relationship between the observed host range of diaspidid species and their abundance on each individual host. Here we plot every tree colonized by each diaspidid species independently, and dot 
area is proportional to the number of data points at that coordinate. Results are divided by location and host taxonomic level: a) Panama, species; b) Panama, genus; c) Panama, family; d) Malaysia, species; e) Malaysia, genus; f) Malaysia, family. None of these relationships (as fitted by a linear model, dashed line) was statistically different from expectations under a null model (all $P>0.9)$.
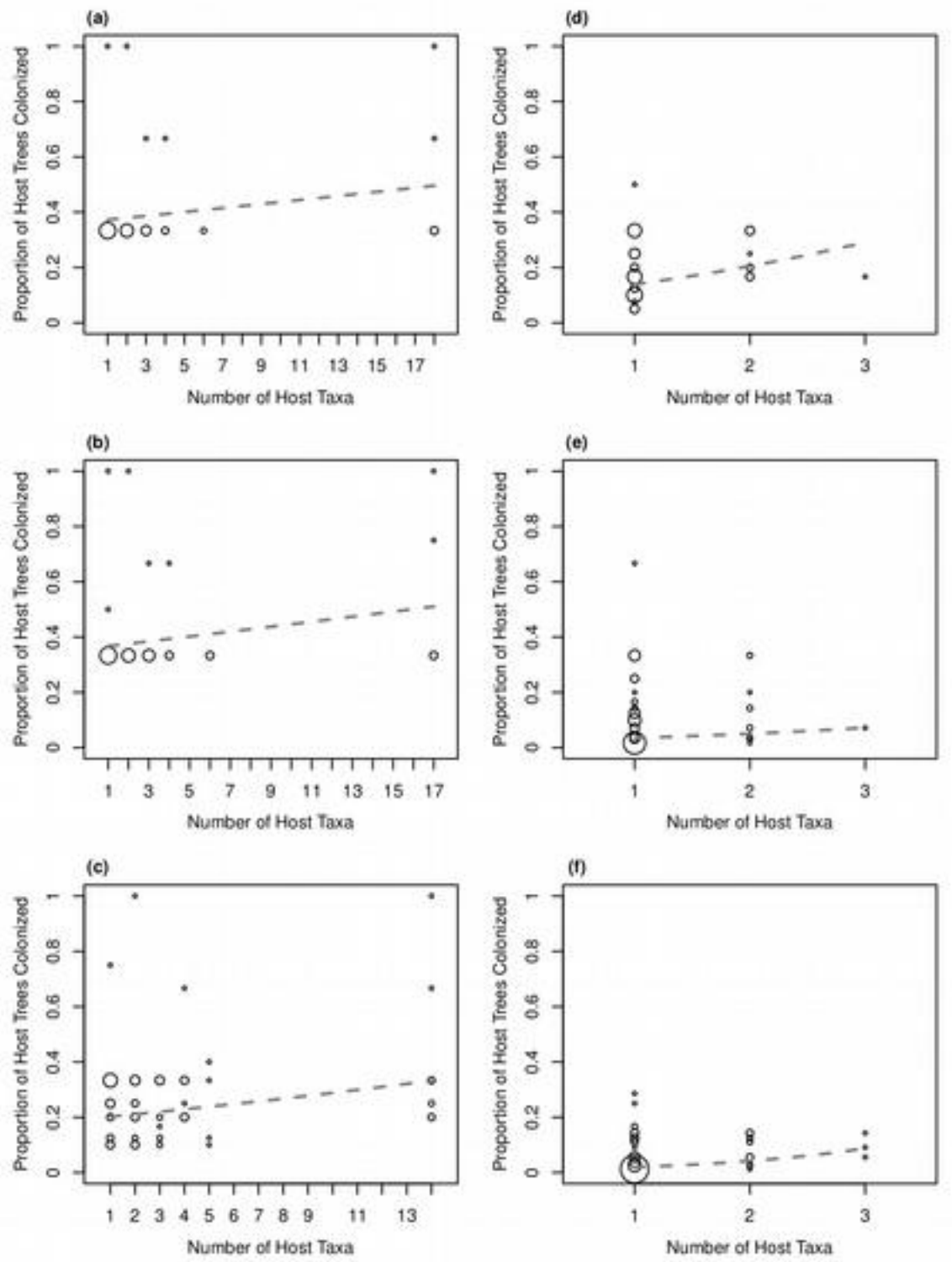

Figure 2. Diaspidid species with larger host ranges were present on a higher proportion of the individual trees in their host range. Here each observed host-taxon-by-diaspidid-species interaction is plotted independently, although host taxa with fewer than three tree individuals surveyed were excluded from this analysis. Circle area is proportional to the number of data points at that coordinate. Results are divided by location and host taxonomic level: a) Panama, species; b) Panama, genus; c) Panama, family; d) Malaysia, species; e) Malaysia, genus; f) Malaysia, family. All fitted slopes (dashed lines) were positive and all were statistically significant $(P<0.05)$, except in Malaysia by host genus $(P=0.062)$.

\section{Literature Cited}


Ali, J. G., and A. A. Agrawal. 2012. Specialist versus generalist insect herbivores and plant defense. Trends Plant Sci. 17:293-302.

Andersen, J. C., J. Wu, M. E. Gruwell, R. Gwiazdowski, S. E. Santana, N. M. Feliciano, G. E. Morse, and B. B. Normark. 2010. A phylogenetic analysis of armored scale insects (Hemiptera: Diaspididae), based upon nuclear, mitochondrial, and endosymbiont gene sequences. Mol. Phylogenet. Evol. 57:992-1003.

Barrett, L. G., and M. Heil. 2012. Unifying concepts and mechanisms in the specificity of plant-enemy interactions. Trends Plant Sci. 17:282-292.

Benjamini, Y., and Y. Hochberg. 1995. Controlling the false discovery rate: a practical and powerful approach to multiple testing. J. R. Stat. Soc. Ser. B 57:289-300.

Bernays, E. A. 2001. Neural limitations in phytophagous insects: implications for diet breadth and evolution of host affiliation. Annu. Rev. Entomol. 46:703-727.

Capella-Gutiérrez, S., J. M. Silla-Martínez, and T. Gabaldón. 2009. trimAl: a tool for automated alignment trimming in large-scale phylogenetic analyses. Bioinformatics 25:1972-1973.

Celorio-Mancera, M. de la P., C. W. Wheat, M. Huss, F. Vezzi, R. Neethiraj, J. Reimegård, S. Nylin, and N. Janz. 2016. Evolutionary history of host use, rather than plant phylogeny, determines gene expression in a generalist butterfly. BMC Evol. Biol. 16:59. BMC Evolutionary Biology.

Dixon, A. F. G. 2005. Insect herbivore-host dynamics: tree-dwelling aphids. Cambridge University Press, Cambridge, 199pp.

Egan SP, Ragland GJ, Assour L, Powell TH, Hood GR, Emrich S, Nosil P, Feder JL. 2015. Experimental evidence of genome wide impact of ecological selection during early stages of speciation with gene flow. Eco. Let. 18(8): 817-825

Forister, M. L., L. A. Dyer, M. S. Singer, J. O. Stireman, and J. T. Lill. 2012. Revisiting the evolution of ecological specialization, with emphasis on insect-plant interactions. Ecology 93:981-991.

Forister, M. L., and S. H. Jenkins. 2017. A neutral model for the evolution of diet breadth. Am. Nat. 190:E40-E54.

Forister, M. L., V. Novotny, A. K. Panorska, L. Baje, Y. Basset, P. T. Butterill, L. Cizek, P. D. Coley, F. Dem, I. R. Diniz, P. Drozd, M. Fox, A. E. Glassmire, R. Hazen, J. Hrcek, J. P. Jahner, O. Kaman, T. J. Kozubowski, T. A. Kursar, O. T. Lewis, J. Lill, R. J. Marquis, S. E. Miller, H. C. Morais, M. Murakami, H. Nickel, N. A. Pardikes, R. E. Ricklefs, M. S. Singer, A. M. Smilanich, J. O. Stireman, S. Villamarín-Cortez, S. Vodka, M. Volf, D. L. Wagner, T. Walla, G. D. Weiblen, and L. A. Dyer. 2015. The global distribution of diet breadth in insect herbivores. Proc. Natl. Acad. Sci. U. S. A. 112:442-7.

Funk, D. J., D. J. Futuyma, G. Orti, and A. Meyer. 1995. A history of host associations and evolutionary diversification for Ophraella(Coleoptera: Chrysomelidae): new evidence from mitochondrial DNA. Evolution 49:1008-1017.

Futuyma, D. J. 2010. Evolutionary constraint and ecological consequences. Evolution 64:1865-84.

Futuyma, D. J. 2008. Sympatric speciation: norm or exception? Pp. 136-147 in Specialization, Speciation, and Radiation: The Evolutionary Biology of Herbivorous Insects.

Futuyma, D. J., M. Keese, and D. J. Funk. 1995. Genetic constraints on macroevolution: the evolution of host affiliation in the leaf beetle genus Ophraella. Evolution 49:797-809.

Futuyma, D. J., and G. Moreno. 1988. The evolution of ecological specialization. Annu. Rev. Ecol. Syst. 19:207-233.

Gilbert, G. S., and C. O. Webb. 2007. Phylogenetic signal in plant pathogen-host range. Proc. Natl. Acad. Sci. U. S. A. 104:4979-83. 
Gompert, Z., J. P. Jahner, C. F. Scholl, J. S. Wilson, L. K. Lucas, V. Soria-Carrasco, J. A. Fordyce, C. C. Nice, C. A. Buerkle, and M. L. Forister. 2015. The evolution of novel host use is unlikely to be constrained by trade-offs or a lack of genetic variation. Mol. Ecol. 24:2777-2793.

Gwiazdowski, R. A., I. M. Vea, J. C. Andersen, and B. B. Normark. 2011. Discovery of cryptic species among North American pine-feeding Chionaspis scale insects (Hemiptera: Diaspididae). Biol. J. Linn. Soc. 104:47-62.

Gyllenberg, M., and J. Metz. 2001. On fitness in structured metapopulations. J. Math. Biol. 43:545-60.

Hadfield, J. D., and S. Nakagawa. 2010. General quantitative genetic methods for comparative biology: phylogenies, taxonomies and multi-trait models for continuous and categorical characters. J. Evol. Biol. 23:494-508.

Hardy, N. B. 2018. The Biodiversity of Sternorrhyncha: Scale insects, aphids, psyllids, and whiteflies. Insect Biodiversity: Science and Society, pp.591-625.

Hardy, N. B., and S. P. Otto. 2014. Specialization and generalization in the diversification of phytophagous insects: tests of the musical chairs and oscillation hypotheses. Proceedings of the Royal Society B - Biological Sciences 281: 20132960 http://dx.doi.org/10.1098/rspb.2013.2960.

Hardy, N. B., D. A. Peterson, and B. B. Normark. 2016. Nonadaptive radiation: pervasive diet specialization by drift in scale insects? Evolution 70:2421-2428.

Heard, S. B., J. O. Stireman, J. D. Nason, G. H. Cox, C. R. Kolacz, and J. M. Brown. 2006. On the elusiveness of enemy-free space: spatial, temporal, and host-plant-related variation in parasitoid attack rates on three gallmakers of goldenrods. Oecologia, 150:421-434.

Hill, M. G., and T. Holmes. 2009. An analysis of latania scale (Hemiberlesia lataniae) crawler settlement behaviour on kiwifruit leaves and bark. New Zeal. Plant Prot. 62:56-62.

Hogenhout, S. A., and J. I. B. Bos. 2011. Effector proteins that modulate plant-insect interactions. Curr. Opin. Plant Biol. 14:422-428.

Hutchinson, G. E. 1959. Homage to Santa Rosalia or why are there so many kinds of animals? Am. Nat. 93:145-159.

Janz, N., K. Nyblom, and S. Nylin. 2001. Evolutionary dynamics of host-plant specialization: a case study of the tribe Nymphalini. Evolution 55:783-796.

Joshi, A., and J. N. Thompson. 1995. Trade-offs and the evolution of host specialization. Evol. Ecol. 9:82-92.

Kelley, S. T., and B. D. Farrell. 1998. Is specialization a dead end? The phylogeny of host use in Dendroctonus bark beetles (Scolytidae). Evolution 52:1731-1743.

Krasnov, B. R., M. A. Fortuna, D. Mouillot, I. S. Khokhlova, G. I. Shenbrot, and R. Poulin. 2012. Phylogenetic signal in module composition and species connectivity in compartmentalized host-parasite networks. Am. Nat. 179:501-511.

Magsig-Castillo, J., J. G. Morse, G. P. Walker, J. L. Bi, P. F. Rugman-Jones, and R. Stouthamer. 2010. Phoretic dispersal of armored scale crawlers (Hemiptera: Diaspididae). J. Econ. Entomol. 103:1172-1179.

Mirarab, S., N. Nguyen, and T. Warnow. 2014. PASTA: ultra-large multiple sequence alignment. Res. Comput. Mol. Biol. 177-191.

Neher, R. A. 2013. Genetic draft, selective interference, and population genetics of rapid adaptation. Ann. Rev. Eco. Evo. Sys. 44:195-215. 
Normark, B. B., G. E. Morse, A. Krewinski, and A. Okusu. 2014. Armored scale insects (Hemiptera: Diaspididae) of San Lorenzo National Park, Panama, with descriptions of two new species. Ann. Entomol. Soc. Am. 107:37-49.

Normark, B. B., and N. A. Johnson. 2011. Niche explosion. Genetica 139:551-564.

Nurmi, T. and K. Parvinen, 2008. On the evolution of specialization with a mechanistic underpinning in structured metapopulations. Theor. Pop. Bio. 73:22-3243.

Peterson, D. A., N. B. Hardy, G. E. Morse, I. C. Stocks, A. Okusu, and B. B. Normark. 2015. Phylogenetic analysis reveals positive correlations between adaptations to diverse hosts in a group of pathogen-like herbivores. Evolution 69:2785-2792.

Peterson, D. A., N. B. Hardy, and B. B. Normark. 2016. Micro- and macroevolutionary trade-offs in plant-feeding insects. Am. Nat. 188:640-650.

R Core Team. 2017. R: A language and environment for statistical computing. R Foundation for Statistical Computing, Vienna, Austria.

Ravigne, V., U. Dieckmann, and I. Olivieri. 2009. Live where you thrive: joint evolution of habitat choice and local adaptation facilitates specialization and promotes diversity. Am. Nat. 174:E141-E169.

Remold, S. 2012. Understanding specialism when the jack of all trades can be the master of all. Proc. R. Soc. B Biol. Sci. 279:4861-4869.

Rodriguez-Verdugo, A., D. Carrillo-Cisneros, A. Gonzalez-Gonzalez, B. S. Gaut, and A. F. Bennett. 2014. Different tradeoffs result from alternate genetic adaptations to a common environment. Proc. Natl. Acad. Sci. 111:12121-12126.

Singer, M. S., and J. O. Stireman. 2005. The tri-trophic niche concept and adaptive radiation of phytophagous insects. Ecol. Lett. 8:1247-1255.

Smith, S. A., and B. C. O'Meara. 2012. treePL: divergence time estimation using penalized likelihood for large phylogenies. Bioinformatics 28:2689-2690.

Stamatakis, A. 2014. RAxML version 8: a tool for phylogenetic analysis and post-analysis of large phylogenies. Bioinformatics 30:1312-3.

Vea, I. M., and D. A. Grimaldi. 2016. Putting scales into evolutionary time: the divergence of major scale insect lineages (Hemiptera) predates the radiation of modern angiosperm hosts. Sci. Rep. 6:23487. Nature Publishing Group.

\section{Data Accessibility}

DNA sequences will be deposited in GenBank, and trophic link data and analysis scripts will be uploaded to Dryad upon acceptance.

\section{Competing Interests}

The authors are unaware of any conflicts of interest.

Author ContributionsBBN, GEM, and DAP designed the study. All authors except NBH obtained the data. DAP and NBH analyzed the data and wrote the paper, with input from BBN, GEM, JW, and TI. 